\title{
On the stability of ocean overflows
}

\author{
LARRY J. PRATT ${ }^{1} \dagger, K A R L$ R. HELFRICH ${ }^{1}$ \\ AND DAVID LEEN ${ }^{2}$ \\ ${ }^{1}$ Woods Hole Oceanographic Institution, 360 Woods Hole Rd., Woods Hole, MA 02543, USA \\ ${ }^{2}$ School of Mathematics, Trinity College, Dublin, Ireland
}

(Received 15 March 2007 and in revised form 24 January 2008)

The stability of a hydraulically driven sill flow in a rotating channel with smoothly varying cross-section is considered. The smooth topography forces the thickness of the moving layer to vanish at its two edges. The basic flow is assumed to have zero potential vorticity, as is the case in elementary models of the hydraulic behaviour of deep ocean straits. Such flows are found to always satisfy Ripa's necessary condition for instability. Direct calculation of the linear growth rates and numerical simulation of finite-amplitude behaviour suggests that the flows are, in fact, always unstable. The growth rates and nonlinear evolution depend largely on the dimensionless channel curvature $\kappa=2 \alpha g^{\prime} / f^{2}$, where $2 \alpha$ is the dimensional curvature, $g^{\prime}$ is the reduced gravity, and $f$ is the Coriolis parameter. Very small positive (or negative) values of $\kappa$ correspond to dynamically wide channels and are associated with strong instability and the breakup of the basic flow into a train of eddies. For moderate or large values of $\kappa$, the instability widens the flow and increases its potential vorticity but does not destroy its character as a coherent stream. Ripa's condition for stability suggests a theory for the final width and potential vorticity that works moderately well. The observed and predicted growth in these quantities are minimal for $\kappa \geqslant 1$, suggesting that the zero-potential-vorticity approximation holds when the channel is narrower than a Rossby radius based on the initial maximum depth. The instability results from a resonant interaction between two waves trapped on opposite edges of the stream. Interactions can occur between two Kelvin-like frontal waves, between two inertia-gravity waves, or between one wave of each type. The growing disturbance has zero energy and extracts zero energy from the mean. At the same time, there is an overall conversion of kinetic energy to potential energy for $\kappa>0$, with the reverse occurring for $\kappa<0$. When it acts on a hydraulically controlled basic state, the instability tends to eliminate the band of counterflow that is predicted by hydraulic theory and that confounds hydraulic-based estimates of volume fluxes in the field. Eddy generation downstream of the controlling sill occurs if the downstream value of $\kappa$ is sufficiently small.

\section{Introduction}

Idealized models of rotating channel flow (e.g. Whitehead, Leetma \& Knox 1974; Gill 1977; Borenäs \& Lundberg 1986, 1988; Killworth 1992) have provided a foundation for the understanding of hydraulic control, upstream influence, and other hydraulic processes thought to occur in deep ocean straits and overflows. 


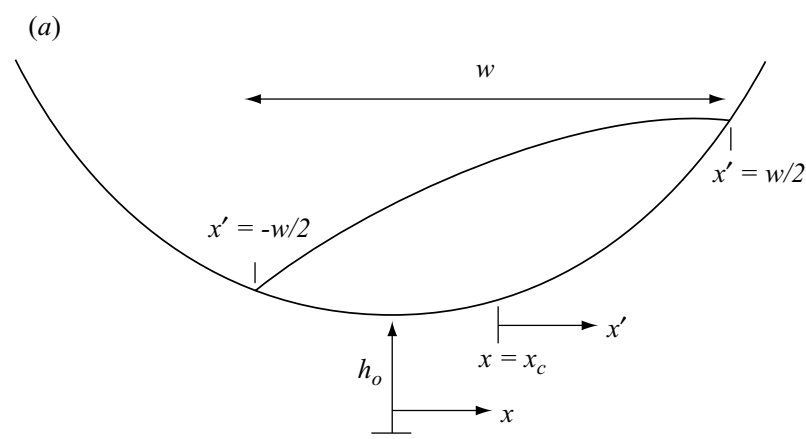

(b)

(c)
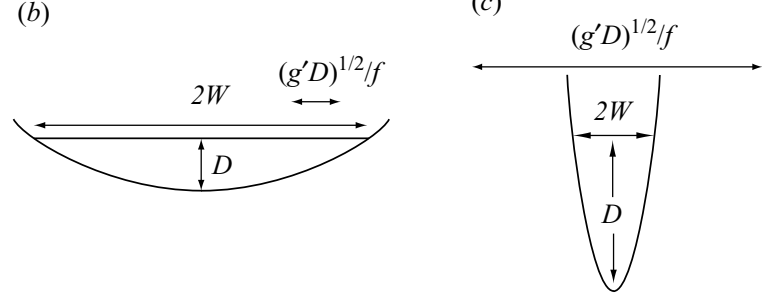

Figure 1. (a) Cross-section of the basic flow. (b) A wide channel. (c) A narrow channel.

These models also provide formulae that relate the volume flux to the upstream conditions (Pratt \& Whitehead 2008). All such models assume steady flow and most are subject to other idealizations deemed necessary for tractability. These include neglect of friction, restriction to uniform potential vorticity, and/or use of channels with rectangular cross-sections. The most widely cited models, at least where volume flux formulae are concerned, are those based on zero-potential-vorticity flow (e.g. Whitehead et al. 1974 and Borenäs \& Lundberg 1988). These models provide the primary motivation for our work. The assumption of zero potential vorticity has the virtue that it can be rationalized by a simple physical argument (see §2) in which fluid columns from a quiescent upstream state are severely squashed as they climb up and over a sill. The cross-sectional depth and velocity profiles are particularly simple and the along-channel structure forms the basis for textbook examples of rotating hydraulic behavior. In addition, it is has been shown by Paldor (1983) that zero-potential-vorticity flows are stable when they are confined to a channel with a rectangular cross-section, as assumed in most cases. This stability seems to be reflected in laboratory and numerical experiments (e.g. Whitehead et al. 1974; Shen 1981; Pratt, Helfrich \& Chassignet 2000; Helfrich \& Pratt 2003) where conditions of uniform potential vorticity are approximated and the cross-section is rectangular. On the other hand, the work of Griffiths, Killworth \& Stern (1983, hereafter GKS), has shown that a dense bottom current with zero potential vorticity is generally unstable when it flows along a flat bottom with a constant cross-stream slope. The stream is no longer confined by vertical sidewalls but has edges (or fronts) that are free to wander up and down the slope. The primary instability is associated with a resonant interaction between two waves that are trapped on the opposite edges of the stream.

The real situation for deep ocean strait and sill topography lies somewhere between the above extremes. As suggested in figure 1(a), the flows are confined to a channel, but with a smooth, rounded shape that allows the thickness of the deep layer to 
go to zero at the edges. The edges are free to meander but still somewhat confined by the topography. The stability of such flows is plausibly tied to the curvature of the bottom: large curvature ostensibly restricts transverse motion and leads to stability. Given the popularity and importance of zero-potential-vorticity models, it is natural to ask whether the flows they predict are actually stable when the channel bottom is curved. The purpose of this work is to investigate any possible linear and nonlinear instability along with the consequences for the basic character of the flow. As we will show, the flows are all formally unstable, though the instability is weak for large bottom curvature (dynamically narrow channels). Under certain conditions, the instability can alter the cross-sectional properties of the flow, including its width and potential vorticity, and create significant departures from what hydraulic theory predicts.

The present work also has possible ramifications for the variability of deep overflows and the generation of eddies. Strong variability with a dominant period of $2-5$ days is present in the Denmark Strait overflow, as recorded in data from moored arrays (e.g. Worthington 1969; Dickson \& Brown 1994). Smith (1976) has attributed this variability to baroclinic instability. Downstream of the sill, the descending portion of the overflow (the outflow or outflow 'plume') can form eddies that are dominantly cyclonic. These eddies exist over the entire water column and their surface signatures can be seen in satellite imagery (Bruce 1995). Numerical simulations (e.g. Spall \& Price 1998; Etling et al. 2000) have suggested a number of generation mechanisms, all of them based on interactions with the overlying water masses. We will show that downstream eddy generation is possible without the need for an active upper layer, provided that the bottom curvature in the downstream region is sufficiently weak.

\section{Overflows with zero potential vorticity and a rounded channel}

We will consider a shallow, homogeneous layer of fluid, flowing in a deep channel. The layer is overlain by an inactive water mass having slightly lower density. The channel is aligned in the $y$-direction and has a rounded cross section, as shown in figure $1(a)$. The elevation of the bottom is given by $h$, the layer thickness by $d$, and the along- and cross-channel velocities by $v$ and $u$, all non-dimensional. In terms of their dimensional (starred) counterparts, the variables are given by

$$
\begin{gathered}
x=\frac{x^{*} f}{\left(g^{\prime} H\right)^{1 / 2}}, \quad y=\frac{y^{*}}{L}, \quad t=\frac{t^{*}\left(g^{\prime} H\right)^{1 / 2}}{L}, \\
v=\frac{v^{*}}{\left(g^{\prime} H\right)^{1 / 2}}, \quad u=\frac{f L u^{*}}{g^{\prime} H}, d=\frac{d^{*}}{H}, h=\frac{h^{*}}{H},
\end{gathered}
$$

where $H$ and $L$ are unspecified depth and along-channel length scales and $g^{\prime}$ is the reduced gravity.

The deep stream is governed by the shallow water equations, non-dimensionally

$$
\begin{gathered}
\delta^{2}\left(\frac{\partial u}{\partial t}+u \frac{\partial u}{\partial x}+v \frac{\partial u}{\partial y}\right)-v=-\frac{\partial d}{\partial x}-\frac{\partial h}{\partial x}, \\
\frac{\partial v}{\partial t}+u \frac{\partial v}{\partial x}+v \frac{\partial v}{\partial y}+u=-\frac{\partial d}{\partial y}-\frac{\partial h}{\partial y} \\
\frac{\partial d}{\partial t}+\frac{\partial(u d)}{\partial x}+\frac{\partial(v d)}{\partial y}=0 .
\end{gathered}
$$


Use of the full shallow-water equations, rather than 'balance' equations or other approximations, is required in order that hydraulic effects are faithfully captured. The following analysis thereby differs from that of other systems (e.g. Swaters 1991) that have strong frontal features but nearly balanced velocity fields. The parameter $\delta=\left(g^{\prime} H\right)^{1 / 2} / f L$, the ratio of the expected cross-channel length scale $\left(g^{\prime} H\right)^{1 / 2} / f$ and the along-channel length scale, is taken to be $\ll 1$ in hydraulic models and will apply to the gradually varying hydraulic state whose stability we will wish to examine. However, the unstable waves that may grow on this background state will generally have $\delta=O(1)$.

Conservation of potential vorticity, $\mathrm{d} q / \mathrm{d} t=0$, with

$$
q=\frac{1+\partial v / \partial x-\delta^{2} \partial u / \partial y}{d},
$$

follows from the above set. If the flow originates from a relatively deep and quiescent upstream basin of depth $d_{\infty}$, where $q \approx 1 / d_{\infty}$, then the severe squashing of fluid columns that accompanies motion into a much shallower channel implies that $1+$ $\partial v / \partial x \approx d / d_{\infty} \ll 1$. The 'zero' potential vorticity approximation

$$
\frac{\partial v}{\partial x}=-1
$$

then applies to these shallow reaches.

In much of what is to come, the deep channel will have a parabolic shape:

$$
h^{*}\left(x^{*}, y^{*}\right)=h^{*}\left(0, y^{*}\right)+\alpha\left(y^{*}\right) x^{* 2},
$$

non-dimensionally

$$
h(x, y)=h_{o}(y)+\frac{1}{2} \kappa(y) x^{2} .
$$

Here $\kappa(y)=2 \alpha(y) g^{\prime} / f^{2}$ is a non-dimensional measure of the cross-channel bottom curvature, also a measure of the channel width. This latter can be illustrated by considering the half-width $W$ of the interface when the channel is filled to a centreline depth $D$ with a resting fluid (figure $1 b$ ). The width of the surface is equal to $2(D / \alpha)^{1 / 2}$ for a parabolic channel. For this $D$, rotational effects may be expected to act over the Rossby radius of deformation $L_{d}=\left(g^{\prime} D\right)^{1 / 2} / f$. Thus $\kappa=2 L_{d}^{2} / W^{2}$ and $\kappa \ll 1$ $(\kappa \gg 1)$ corresponds to a dynamically wide (narrow) channel, as shown in figure $1(b)$ (figure 1c).

Zero-potential-vorticity solutions in a parabolic channel of gradually varying curvature and height were first written down by Borenäs \& Lundberg (1988). We will employ the same solutions, formulated in a different variable set. The cross-channel profiles of velocity and depth can be obtained by first substituting (2.6) into (2.1) and setting $\delta=0$, which leads to

$$
v=\frac{\partial d}{\partial x}+\kappa x .
$$

Let $x_{c}(y)$ represent the centreline position of the current and $w(y)$ the width (figure 1a) and introduce the centred coordinate $x^{\prime}=x-x_{c}$. Then the layer thickness and velocity are obtained by combining the above relation with (2.5), leading to

$$
d=\frac{1}{2}(1+\kappa)\left[\left(\frac{1}{2} w\right)^{2}-x^{\prime 2}\right]
$$

and

$$
v=\kappa x_{c}-x^{\prime} .
$$




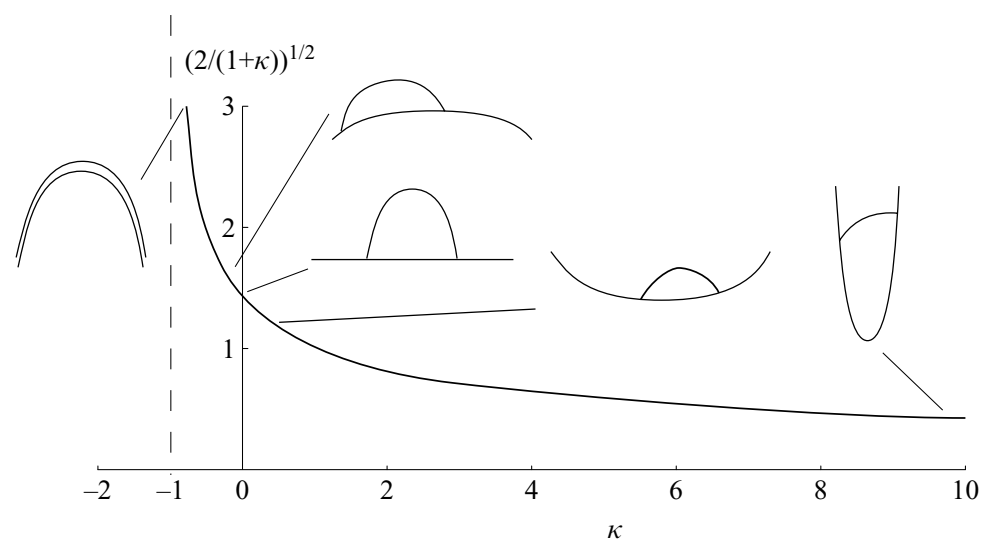

Figure 2. Possible configurations for zero-potential-vorticity flow in a parabolic channel, according to the value of the bottom curvature $\kappa$. The width of the flow scales with the Rossby radius of deformation times the factor $[2 /(1+\kappa)]^{1 / 2}$.

The non-dimensional width of the flow is determined only by $\kappa$ and by the centreline depth. If $(2.8)$ is evaluated at $x^{\prime}=0$ the width is found as $\frac{1}{2} w=(2 /(1+\kappa))^{1 / 2}\left(d_{x^{\prime}=0}\right)^{1 / 2}$ or, in dimensional terms,

$$
\frac{1}{2} w^{*}=\left(\frac{2}{1+\kappa}\right)^{1 / 2}\left(\left.g^{\prime} d^{*}\right|_{x^{\prime}=0}\right)^{1 / 2} / f
$$

Thus, the half-width of the current scales with the Rossby radius of deformation, based on the centreline depth, within a factor $2^{1 / 2} /(1+\kappa)^{1 / 2}$. This factor is plotted in figure 2 over the permissible range of the curvature $-1<\kappa$. If the channel curvature is small $(|\kappa| \ll 1)$ the bottom is essentially flat and the current half-width is approximately $\sqrt{ } 2$ (figure 2 insets.) This is essentially the same case as that considered by GKS. If the channel is highly curved and concave $(\kappa \gg 1)$ then the width is much less than the deformation radius. This case is the closest one can get to a rectangular channel. At the other extreme $(\kappa \rightarrow-1)$, the bottom is convex and the curvature of the free surface approaches that of the bottom. In this case $d \rightarrow 0$ and the bottom is covered with a thin sheet of fluid with infinite width.

The volume flux of the current is

$$
Q=\int_{-w / 2}^{w / 2}(v d) \mathrm{d} x^{\prime}=\frac{\kappa(1+\kappa)}{12} w^{3} x_{c} .
$$

The Bernoulli function $B$, approximated by $v^{2} / 2+d+h$ for the gradually varying flow, is uniform for zero-potential-vorticity flow. Its value can be calculated by evaluation at either edge of the current, leading to

$$
B=\frac{1}{2} v^{2}+d+h=h_{o}+\frac{1}{2}(1+\kappa)\left(\frac{1}{4} w^{2}+\kappa x_{c}^{2}\right)
$$

A single equation for the variable $w$ can be obtained through elimination of $x_{c}$ from the last two relations, resulting in

$$
B=h_{o}+(1+\kappa)\left(\frac{w^{2}}{8}+\frac{72 Q^{2}}{\kappa(1+\kappa)^{2} w^{6}}\right) .
$$

For given $B$ and $Q,(2.12)$ relates specified topographic parameters $h_{o}(y)$ and $\kappa(y),(2.12)$ to the flow variable $w$ at any $y$. We are particularly interested in 


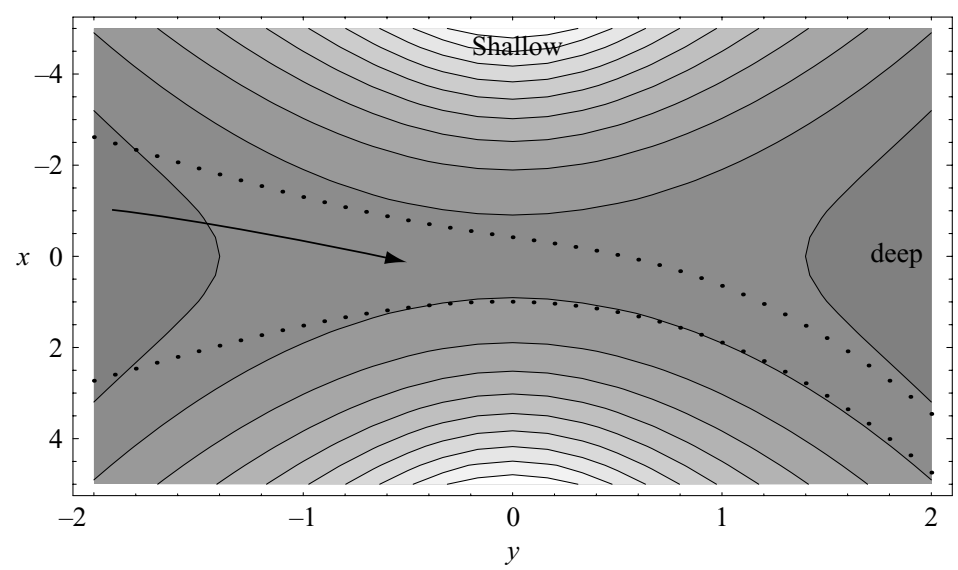

FIGURE 3. The dotted lines show the edges of a hydraulically controlled current with zero potential vorticity and $B=1$, flowing across a sill separating two deep basins. The topography is given by $h_{o}(y)=-y^{2}$ and $\kappa(y)=2 /\left(1+y^{2}\right)$. The net volume flux is to the right. Darker shades indicate deeper regions of the bottom.

hydraulically controlled flows; that is, flows that become hydraulically critical at the most constricted section. The expression on the right-hand side of (2.12) takes the form of the hydraulic function, as defined by Gill (1977), in the variable $w^{2}$. The critical condition for the flow is therefore obtained by setting the derivative (with respect to $w^{2}$ ) of the right-hand side to zero. The result

$$
\frac{w^{2}}{12}=\kappa x_{c}^{2}
$$

is equivalent to that derived by Borenäs \& Lundberg (1988).

We will consider two classes of stability problems for a zero-potential-vorticity basic flow. In the first (more traditional) case, the basic flow is parallel and is confined to a channel that is uniform in $y$. In the second case the basic flow is hydraulically controlled and is confined to a gradually varying channel with a sill (maximum in $h_{o}(y)$ ) and width contraction (maximum in $\kappa(y)$ ). An example of a hydraulically controlled solution with $B=1$, flowing between two deep basins, is shown in figure 3 . The topography is determined by (2.6) with $h_{o}(y)=-y^{2}$ and $\kappa(y)=2 /\left(1+y^{2}\right)$.

\section{Energetics for parallel basic states}

For the case of a uniform channel we denote by $v=V(x)$ and $d=D(x)$ the parallel basic state whose stability is to be examined. We will temporarily allow the bottom topography and potential vorticity to be arbitrary. The following discussion is largely restricted to flow instability with respect to discrete wave modes. It is well known that non-modal disturbances to flow with shear can experience temporary growth if the initial perturbation is aligned favourably. This situation will not be considered, but the interested reader can consult Farrell \& Ioannou (1996) and references contained therein for more details.

Instability is traditionally defined and measured in terms of the growth in time of some positive definite quantity, sometimes a wave energy norm (other norms may be used, including enstrophy). The wave draws on kinetic energy that is available in the mean ( $y$-average) state due to horizontal shear, and/or on potential energy 
associated with gradients in the mean interface elevation. As the wave energy grows, the energy contained in the mean fields diminishes. For the shallow-water models used in hydraulics, in which Poincare and Kelvin waves, and their relatives, are permitted, the energy associated with the wave is no longer positive definite. As discussed by Hayashi \& Young (1987), the notion that the wave draws energy from the mean flow must be re-examined.

Suppose that the disturbed flow is periodic or isolated in $y$. Let $A$ represent a rectangular, horizontal region that completely encloses the wetted area of the flow and extends over a spatial period in the $y$-direction. Conservation of energy for the fluid over $A$ can be derived ${ }^{\dagger}$ from (2.1)-(2.3) in the form

$$
\mathrm{d} E / \mathrm{d} t=0
$$

where

$$
E=\frac{1}{2} \iint_{A}\left[d\left(u^{2}+v^{2}\right)+d^{2}+2 d h\right] \mathrm{d} \sigma
$$

and $\mathrm{d} \sigma$ is the elemental area. Note that $\delta$ is now regarded as unity since the flow may contain disturbances of small scale in $y$. Next, separate the flow into its basic (parallel) part $(V, D)$ and a residual, or 'disturbance', that has evolved from an initial perturbation of amplitude $\varepsilon \ll 1$. We will consider the energetics of the flow during the early stage in which a growing disturbance has not exceeded this magnitude. The disturbance is then represented as an $\varepsilon$-expansion and the complete flow field is given by

$$
\left.\begin{array}{rl}
v & =V(x)+\varepsilon v_{0}+\varepsilon^{2} v_{1}+\cdots, \\
u & =\varepsilon u_{0}+\varepsilon^{2} u_{1}+\cdots, \\
d & =D(x)+\varepsilon d_{0}+\varepsilon^{2} d_{1}+\cdots, \\
q & =Q(x)+\varepsilon q_{0}+\varepsilon^{2} v_{1}+\cdots,
\end{array}\right\}
$$

The lowest-order disturbance fields $v_{0}, d_{0}$, etc. satisfy the linearized shallow-water equations:

$$
\begin{gathered}
\left(\frac{\partial}{\partial t}+V \frac{\partial}{\partial y}\right) u_{0}-v_{0}=-\frac{\partial d_{0}}{\partial x}, \\
\left(\frac{\partial}{\partial t}+V \frac{\partial}{\partial y}\right) v_{0}+Q D u_{0}=-\frac{\partial d_{0}}{\partial y}, \\
\left(\frac{\partial}{\partial t}+V \frac{\partial}{\partial y}\right) d_{0}+\frac{\partial\left(D u_{0}\right)}{\partial x}+\frac{\partial\left(D v_{0}\right)}{\partial y}=0 \\
\left(\frac{\partial}{\partial t}+V \frac{\partial}{\partial y}\right) q_{0}+u_{0} \frac{\partial Q}{\partial x}=0
\end{gathered}
$$

Here

$$
Q=\frac{1+\partial V / \partial x}{D}
$$

is the basic-state potential vorticity and

$$
q_{0}=D^{-1}\left(\frac{\partial v_{0}}{\partial x}-\frac{\partial u_{0}}{\partial y}-Q d_{0}\right)
$$

is the perturbation potential vorticity.

$\dagger$ In performing this and other derivations, it may be helpful to think of the non-wetted portion of $A$ to be covered by a thin film of water. 
Under conditions of growth, the mean ( $y$-average) components of the flow may alter. These disturbance means first arise within the fields $v_{1}, d_{1}$, as determined at a higher order of approximation. Following Hayashi \& Young (1987), it is helpful to separate the total energy into three parts: $E=E_{b}+E_{w}+E_{m}+O\left(\varepsilon^{3}\right)$. $E_{b}$ is the energy associated with the basic flow and is a constant. $E_{m}$ is the energy associated with the aforementioned mean fields. The 'wave' energy $E_{w}$, defined by

$$
E_{w}=\frac{\varepsilon^{2}}{2} \iint_{A}\left[D\left(u_{0}^{2}+v_{0}^{2}\right)+d_{0}^{2}+2 V v_{0} d_{0}\right] \mathrm{d} \sigma,
$$

is the energy associated with the wavy perturbation and is composed of the integrals of the corresponding quadratic quantities in the perturbation fields. In quasi-geostrophic or two-dimensional systems, the wave energy consists of just the first three terms in the integrand, all of which are positive definite. Shallow-water dynamics require one to include the fourth term, which is not sign definite. The presence of this term is the key to special energetic properties of the problem at hand.

Since total energy is conserved, the sum $E_{w}+E_{m}$ must also be conserved, at least to $O\left(\varepsilon^{3}\right)$, as an instability develops. If the wave grows by extracting energy from the mean flow, $E_{w}$ increases and $E_{m}$ decreases. The growth of $E_{w}$ is associated with a potential vorticity flux, as shown by the relation

$$
\frac{\partial}{\partial t}\left[E_{w}+\iint_{A} D^{2} V \frac{\eta^{2}}{2} \frac{\partial Q}{\partial x} \mathrm{~d} \sigma\right]=0,
$$

which can be derived from $(3.3 a-c)$. Here $\eta(x, t)$ represents the lateral displacement of a fluid parcel away from its equilibrium position in the basic state. For uniform potential vorticity, $\partial Q / \partial x=0$, the potential vorticity flux is zero, and $E_{w}$ remains constant. $E_{m}$ must then also remain constant. In a quasi-geostrophic system, where $E_{w}$ is composed entirely of positive definite terms $u_{0}^{2}, d_{0}^{2}$, etc., conservation of $E_{w}$ rules out instability. In shallow water, the presence of the additional, potentially negative, term $2 V u_{0} v_{0}$ means that the $u_{0}$, $v_{0}$, etc. can amplify while wave energy is preserved. Similar statements may be made with respect to momentum. Under these conditions it is incorrect to think of the wave as drawing energy from the mean flow. The mean may change, but these changes must occur in a way that leaves $E_{m}$ fixed.

For an amplifying wave, the values of $E_{w}$ and $E_{m}$ must not only remain constant, these constants must be zero. This follows from the fact that the disturbance can be traced backwards in time and thereby be made arbitrarily small. For example, an exponentially growing disturbance will have $E_{w}=A \mathrm{e}^{2 l c_{i} t}$ for some coefficient $A$. But since $E_{w}$ is constant, the coefficient $A$ must be zero. On the other hand, neutral waves may have fixed, finite values of $E_{w}$ and $E_{m}$.

These ideas are discussed further by Hayashi \& Young (1987) in connection with an equatorial flow. Application to the present system requires only that (3.7) be shown to remain valid in the presence of variable topography. This is easily done but is not proved here. The importance of this discussion for hydraulics is that instabilities that potentially occur within most fundamental hydraulic models, including Whitehead et al. (1974), Gill (1977) and Borenäs \& Lundberg (1986, 1988), do not draw energy from the mean and have zero wave energy. It is therefore difficult to classify any such instabilities as 'barotropic' or 'baroclinic'. This finding does not rule out the possibility that there can be an overall conversion of total potential energy to kinetic energy, or vice-versa, as the disturbance evolves. This, in fact, is exactly what occurs in the present problem. 


\section{Ripa's theorem and hydraulics}

The familiar necessary conditions for instability of barotropic or quasi-geostrophic flows due to Rayleigh (1880), Kuo (1949), and Charney \& Stern (1962) do not apply to the shallow-water flow under consideration. The only known condition for stability is due to Ripa (1983). His original derivation assumes a flat bottom, but the result can easily be shown to apply in the arbitrary-cross-sectional channel topography (Pratt \& Whitehead 2008).

Ripa's theorem is usually presented as a sufficient condition for stability. There are two requirements: first, a constant $\gamma$ must exist such that $(V-\gamma)^{2} \leqslant D$, or

$$
-D^{1 / 2} \leqslant V-\gamma \leqslant D^{1 / 2},
$$

for all $x$ across the section in question. If it is also the case that

$$
(V-\gamma) \frac{\partial Q}{\partial x} \geqslant 0
$$

for each $x$, then the flow is stable. The first provision relates to gravity wave propagation while the second, which is identical to Fjøtorft's (1950) condition for stability, relates to potential-vorticity wave propagation.

For the models of rotating channel flow with constant potential vorticity, the second requirement $(4.1 b)$ of Ripa's sufficient condition for stability is satisfied. The first requirement (4.1 $a$ ) is essentially that a frame of reference $\mathrm{d} y / \mathrm{d} t=\gamma$ exists in which the magnitude $|V| / D^{1 / 2}$ of the local Froude number is less than unity at each $x$. A graphical interpretation of this condition can be obtained by plotting the profiles of $\pm D^{1 / 2}$ and $V$ (figure $4 a$ ). The requirement is satisfied if one can uniformly shift the $V$ profile up or down so that it fits within the shaded envelope. If the depth goes to zero at both edges of the stream, the thickness of the envelope goes to zero at the edges (figure $4 b$ ) and the condition is nearly impossible to satisfy. The value of $V$ at the two edges of the stream must be identical, and $\gamma$ must be chosen as that value. With zero potential vorticity, the velocity varies linearly across the channel and the two edge velocities can never be equal. Although some zero-potential-vorticity flows in a rectangular channel with sidewalls are provably stable by Ripa's theorem, no zero-potential-vorticity flow with free edges can be so shown. Indeed, the results of our eigenvalue calculations will suggest that all such flows are unstable, though the instability may be weak and of small scale. For flow with arbitrary potential vorticity and free edges, Ripa's theorem can be satisfied only in the exceptional case that $V$ is identical at the two free edges.

\section{The linear stability problem}

Let

$$
\left(\begin{array}{c}
u_{0} \\
v_{0} \\
d_{0}
\end{array}\right)=\operatorname{Re}\left[\left(\begin{array}{c}
\hat{u}(x) \\
\hat{v}(x) \\
\hat{d}(x)
\end{array}\right) \mathrm{e}^{\mathrm{i} l(y-c t)}\right]+O(\varepsilon) .
$$

Substitution into $(3.3 a-c)$ then leads to

$$
\begin{gathered}
{[i l(V-c) \hat{u}]-\hat{v}=-\frac{\mathrm{d}}{\mathrm{d} x} \hat{d},} \\
\mathrm{i} l(V-c) \hat{v}+\left(1+\frac{\partial V}{\partial x}\right) \hat{u}=-\mathrm{i} l \hat{d},
\end{gathered}
$$



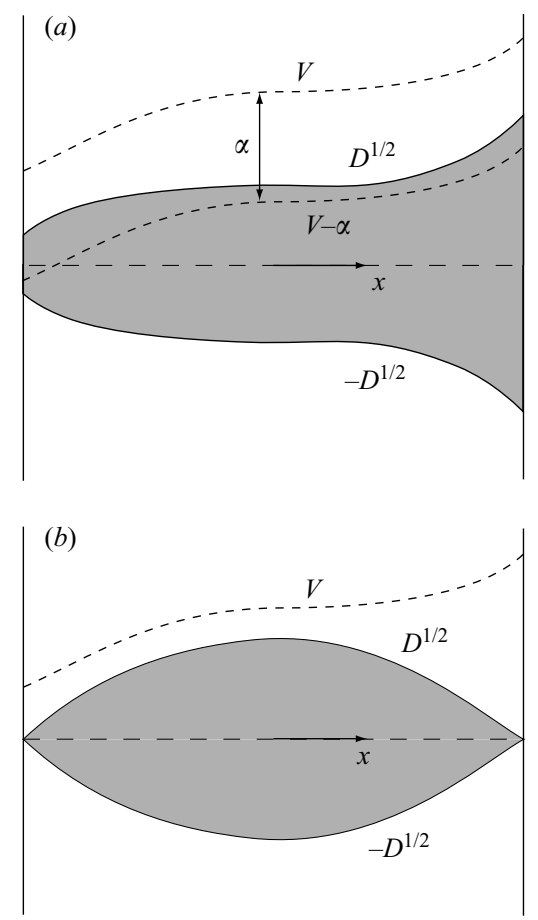

FIGURE 4. Graphical representation of one of the two requirements (see (4.1a)) of Ripa's Theorem. Stability requires that the velocity profile can be shifted uniformly up or down to fit entirely in the shaded area. In $(b)$ the depth goes to zero at the edges of the flow, eliminating the range over which the velocity can be shifted.

$$
\mathrm{i} l(V-c) \hat{d}+\frac{\partial}{\partial x}(D \hat{u})+\mathrm{i} l D \hat{v}=0 .
$$

The boundary conditions are

$$
D \hat{u}=0 \text { (at free edges). }
$$

Since $D$ vanishes at the edges, we merely require that $\hat{u}$ remain bounded there.

There are apparently no general theorems informing solutions to the eigenvalue problem (5.1) and (5.2) with regard to the existence or enumeration of solutions.

For zero potential vorticity $(Q=0),(5.1 b)$ reduces to

$$
(V-c) \hat{v}=-\hat{d} .
$$

Also, the perturbation potential vorticity (see (3.5)) must vanish:

$$
\frac{\mathrm{d} \hat{v}}{\mathrm{~d} x}=\mathrm{i} l \hat{u} .
$$

If these last two relations are used eliminate $\hat{v}$ and $\hat{d}$ from (5.1c), it follows that

$$
\frac{\mathrm{d}}{\mathrm{d} x}\left(D \frac{\mathrm{d} \hat{v}}{\mathrm{~d} x}\right)-l^{2}\left[D-(V-c)^{2}\right] \hat{v}=0,
$$

as shown by GKS. At this point, the shape of the bottom is still arbitrary.

Assuming that $\hat{d}$ remains regular at the edges of the current, the boundary condition (5.2) along with (5.1c) implies that $D \mathrm{~d} \hat{v} / \mathrm{d} x=0$ at the edges. Integration of (5.5) across 
the flow then yields

$$
l^{2} \int_{-w / 2}^{w / 2}\left[D-(V-c)^{2}\right] \hat{v} \mathrm{~d} x=0 .
$$

Hayashi \& Young (1987) have shown that the complex phase speed $c=c_{r}+\mathrm{i} c_{i}$ is bounded by Howard's (1961) semicircle theorem. (The equatorial flow treated by the former has $h=0$ but it is a simple matter to extend the proof to the case of variable $h(x)$.) The result is that $c_{r}$ and $c_{i}$ are constrained by

$$
\left[c_{r}+\frac{1}{2}\left(V_{\max }+V_{\min }\right)\right]^{2}+c_{i}^{2} \leqslant\left[\frac{1}{2}\left(V_{\max }-V_{\min }\right)\right]^{2} ;
$$

that is, the complex phase speed of an unstable wave must fall within a semi-circle, lying in the upper half of the $\left(c_{r}, c_{i}\right)$-plane, centred on the $c_{r}$-axis, and lying between velocity extremes $V_{\min }$ and $V_{\max }$. The value of $c_{r}$ for an unstable wave therefore lies within the range of the basic velocity.

Now consider the specific application to a basic state flowing in a parabolic channel. Stern \& Simeonov (2008) have pointed out that $c_{i}$ depends only on $\kappa$ and not the centreline position $x_{c}$ of the basic flow. A proof that is slightly different from theirs proceeds thus. If (2.8) and (2.9) are substituted into (5.5) and the result expressed in terms of the flow-centred coordinate $x^{\prime}=x-x_{c}$ there follows

$$
\frac{\mathrm{d}}{\mathrm{d} x^{\prime}}\left(D \frac{\mathrm{d} \hat{v}}{\mathrm{~d} x^{\prime}}\right)-l^{2}\left[\frac{1}{2}(1+\kappa)\left[\left(\frac{1}{2} w\right)^{2}-x^{\prime 2}\right]-\left(\kappa x_{c}-c-x^{\prime}\right)^{2}\right] \hat{v}=0 .
$$

Here $x_{c}$ should be interpreted as the (fixed) position of the centreline of the basic flow. According to (2.8) $D(x)$ is independent of $x_{c}$, and the latter then appears only in the expression $\kappa x_{c}-c$. The $c$ value for a basic state centred at $x_{c}$ can be obtained by adding the factor $\kappa x_{c}$ to the $c$ value for the channel-centred $\left(x_{c}=0\right)$ profile with the same width $w$. Since $\kappa x_{c}$ is real, $c_{i}$ (and thus the growth rate $l c_{i}$ ) is independent of $x_{c}$. Shifts in the centreline position of a current with a fixed $D$ profile therefore affect only the real part $c_{r}$ of the phase speed by augmenting $c$ by the amount $\kappa x_{c}$.

\section{Long-wave stability and hydraulic states}

Of particular interest in hydraulics is the stability of long waves. Concepts such as subcritical and supercritical flow are defined in terms of long-wave speeds, assumed to be real. Long-wave instability is therefore most threatening to the preservation of traditional hydraulic behaviour. In order to explore the long-wave limit, let $l \ll 1$ and write

$$
\hat{v}=v^{(0)}+l v^{(1)}+l^{2} v^{(2)}+\cdots
$$

and

$$
c=c_{o}+l c_{1}+l^{2} c_{2}+\cdots .
$$

For simplicity, we will normalize $\hat{v}$ such that its maximum value is unity.

The lowest-order approximations to (5.5) and (5.6) can then be written as

$$
\frac{\mathrm{d}}{\mathrm{d} x^{\prime}}\left(D \frac{\mathrm{d} v^{(0)}}{\mathrm{d} x^{\prime}}\right)=0
$$

and

$$
\int_{-w / 2}^{w / 2}\left[D-\left(V-c_{o}\right)^{2}\right] \mathrm{d} x^{\prime}=0
$$


Integration of the first relation and enforcement of the boundary conditions leads to

$$
v^{(0)}=\text { const. }=1,
$$

in accordance with the normalization. The second relation then yields

$$
c_{0}^{2}-2 c_{0}\langle V\rangle+\left\langle V^{2}\right\rangle-\langle D\rangle=0,
$$

where brackets denote a cross-stream average: $\langle v\rangle=(1 / w) \int_{-w / 2}^{w / 2} v \mathrm{~d} x^{\prime}$. The phase speeds of the two waves are therefore given by

$$
c_{o}=\langle V\rangle \pm\left[\langle V\rangle^{2}-\left\langle V^{2}\right\rangle+\langle D\rangle\right]^{1 / 2} .
$$

Long-wave instability occurs for $\langle V\rangle^{2}-\left\langle V^{2}\right\rangle+\langle D\rangle<0$. For real $c_{o}$, (6.1) suggests the generalized Froude number for zero-potential-vorticity flow:

$$
F_{o}=\frac{\langle V\rangle}{\left[\langle V\rangle^{2}-\left\langle V^{2}\right\rangle+\langle D\rangle\right]^{1 / 2}} .
$$

The flow is subcritical, critical or supercritical according to $F_{o}<1,=1,>1$. These results hold for general bottom topography.

For the parabolic channel profiles (2.8) and (2.9), it follows that

$$
c_{o}=\kappa x_{c} \pm \sqrt{\frac{\kappa}{12}} w .
$$

For real $c_{o}$, the Froude number is

$$
F_{o}=\frac{x_{c}}{w} \sqrt{12 \kappa}
$$

The waves in question are relatives of the Kelvin waves that would exist were the flow confined to a channel with vertical sidewalls. When the topography becomes smooth and the layer depths vanish at the edges, the two Kelvin waves are replaced by two frontal waves. Like their Kelvin wave brethren, the waves are trapped to the edges. Long waves are therefore unstable for $\kappa<0$ and marginally unstable for $\kappa=0$ (the GKS case). $\dagger$ This result suggests that a current that encounters a region of convex bottom topography, such as at a shelf break, may become unstable there with respect to long waves. It also suggests that the flow cannot be controlled, at least not in the usual sense, where the topography is convex.

\section{The effect of channel curvature}

The long-wave results suggest positive curvature as a stabilizing effect on the flow. We test this idea by examining the stability of a sequence of parallel basic states in channels with $\kappa=0.2,0$, and -0.4 (figures $5-10$ ). The channel in this case is uniform in $y$. By choosing the depth scale $H$ so that the centreline depth $D(0)=1$, (2.8) yields $w=2 \sqrt{2 /(1+\kappa)}$. All coefficients in (5.8) now depend only on $\kappa$ and $x_{c}$. Since the growth rate of any unstable waves depends only on the former, one may take $x_{c}=0$. The basic flow is then centred in the channel and the flux $Q=0$. For a given wavenumber $l$, a basic state with the same $\kappa$ but non-zero $x_{c}$ will produce the same linear growth rate as for $x_{c}=0$; the corresponding $c_{r}$ value will increase by

$\dagger$ Although $c$ is real for $\kappa>0$, Borenäs (1988) has shown that a spatial amplification of a long-wave disturbance is possible if the basic flow varies with $y$. 

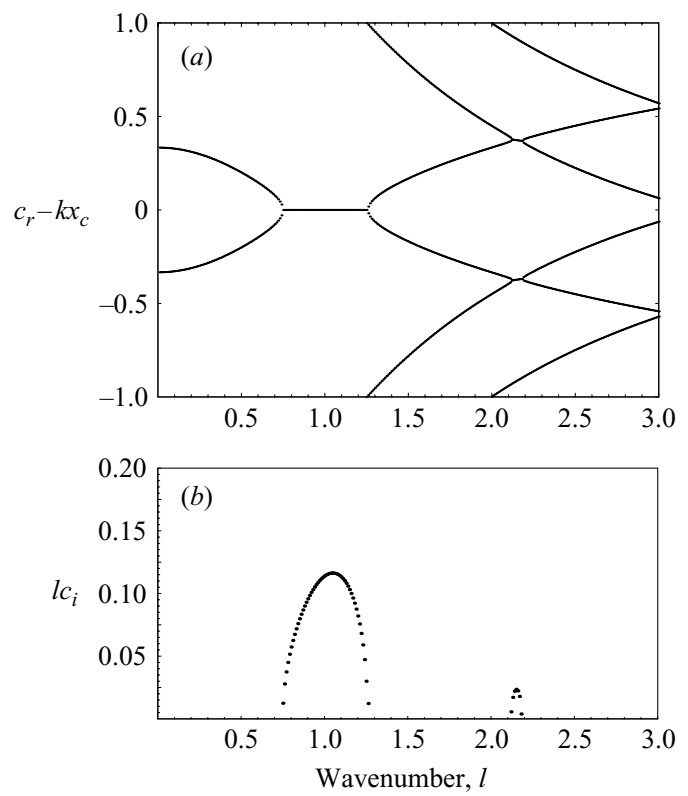

Figure 5. (a) The phase speed (represented by $c_{r}-\kappa x_{c}$ ) and $(b)$ the growth rate $l c_{i}$ as a function of wavenumber $l$ for $\kappa=0.2$.

amount $\kappa x_{c}$. The values of $c_{r}$ and the growth rate $l c_{i}$ are found using a second-order finite-difference approximation of (5.8). In addition, the finite-amplitude evolution of each case is explored using a numerical simulation in which the basic state is subject to an initial random, small-amplitude perturbation in the layer depth. The numerical solution technique uses a second-order finite-volume method for the conservative form of the single-layer shallow-water equations and is described in Helfrich, Kuo \& Pratt (1999). The model has been successfully used in a number of rotating flow problems involving shocks, hydraulic jumps, the presence of zero layer depths and gravity currents (e.g. Pratt et al. 2000). Note that unlike the linear growth stage, the finite-amplitude evolution may depend on the initial $x_{c}$.

We begin with the case $\kappa=0.2$, the linear stability properties of which are plotted in figure 5. At long wavelengths $(l \rightarrow 0)$, the two frontal waves are the only wave modes of the flow. As shown earlier, the phase speeds of the two long waves are distinct, although the corresponding $c_{r}$ curves merge at finite $l \approx 0.75$ (figure $5 a$ ). The resulting instability, which is the result of the interaction between the two frontal waves, generally produces the largest growth rates (figure $5 b$ ). Other wave modes come into play at finite wavelengths. In textbook examples of waves in a prismatic channel with no background flow, these are the Poincare waves. Here we will simply refer to them as inertia-gravity waves. Interactions between edge and inertia-gravity waves, and between different inertial-gravity waves, produce small bands of instability with relatively weak growth rates. The linear analysis for other values of $\kappa$ has shown that the bands of instability migrate towards higher wavenumber as $\kappa$ increases. The growth rates within each band diminish and the flow therefore becomes less unstable in the linear sense.

The finite-amplitude evolution of the perturbed flow for $\kappa=0.2$ reveals a growing meander with a phase shift between the left and right edges of the flow (figure 6). The wavelength is close to that of the most rapidly growing linear disturbance. Eventually 

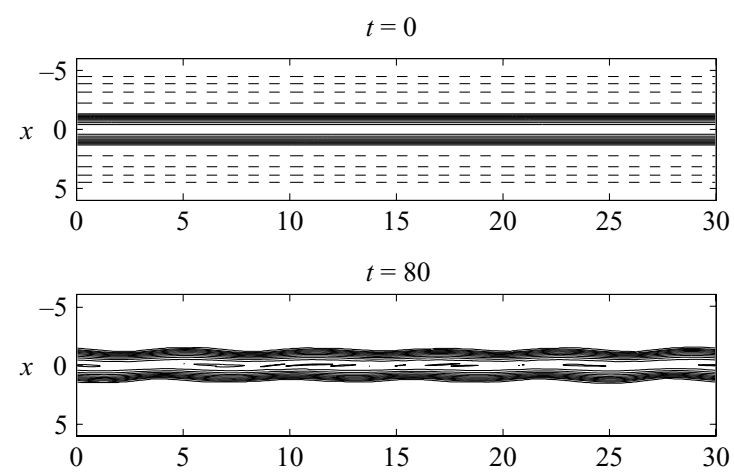

$t=100$

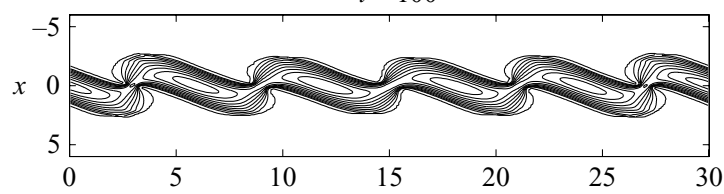

$t=140$

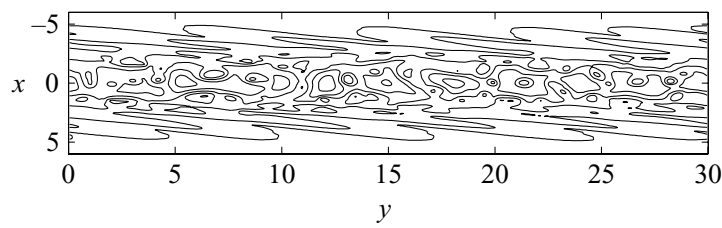

FIGURE 6. The finite-amplitude evolution of a flow with $x_{c}=0$ and $\kappa=0.2$, subjected to a random perturbation. The layer depth is contoured (solid lines) in intervals of 0.1 . The dashed lines in the top panel show the topography contours in intervals of 0.5 . They are not shown in the other panels for clarity.

the waves break and generate mixing around the edges of the flow. The resulting state is a distinct band of current that occupies a width much greater than the initial width $(t=140$ panel).

The case $\kappa=0$ (figure 7) is the essentially the same as that considered by GKS. The long frontal waves now have identical phase speeds and the band of instability, sometimes known as the GKS instability, extends to $l=0$. The maximum growth rate within this band has increased from 0.125 to a value $\approx 0.15$. In addition, the wavenumber range plotted has more bands of instability, each with higher maximum growth rate, than for $\kappa=0.2$. These bands were also identified by Hayashi \& Young (1987) and first recognized in a non-rotating analogue of the present problem by Satomura $(1981 a, b)$. The finite-amplitude evolution for this case (figure 8) again produces phase-shifted meanders, but here the result is the formation of a train of distinct stationary eddies.

The case $\kappa=-0.4$ also has a frontal wave instability band that extends to $l=0$ and there are more bands of higher wavenumber instability (figure 9). The growth rates are larger than for the previous case and the most rapidly growing wave is longer. The finite-amplitude evolution (figure 10) again shows the formation of stationary eddies, this time with a greater along-channel scale.

It has already been shown that an unstable disturbance must have zero wave energy, at least in the early stages of growth. This does not preclude an exchange between the total kinetic and potential energies (as defined by the first two and second 

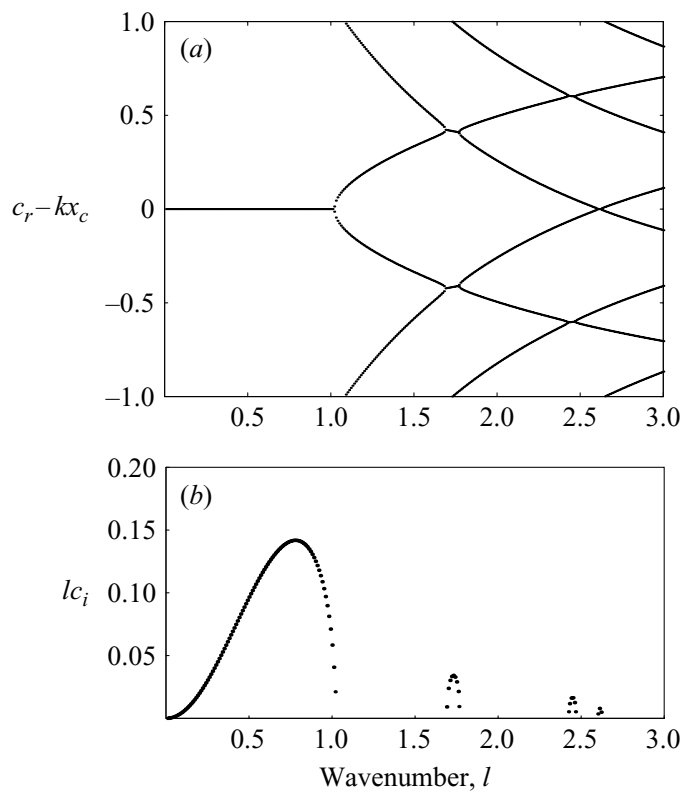

FIGURE 7. Similar to figure 5 but with $\kappa=0$.

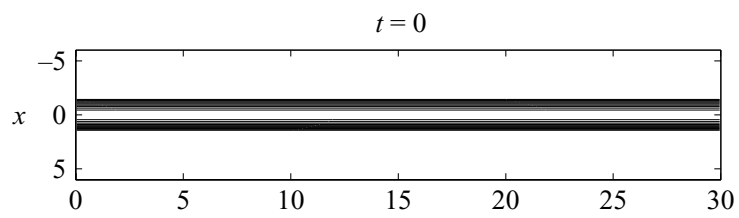

$t=80$
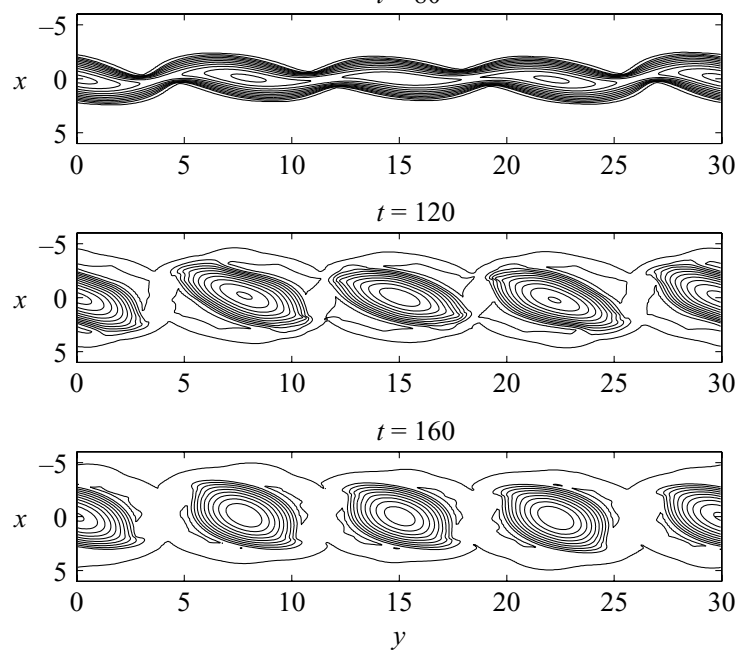

Figure 8. Similar to figure 6 but with $\kappa=0$.

two terms in (3.1)). A history of the total kinetic and potential energy shows a net conversion from kinetic to potential for positive $\kappa$ (figure 11a) and the opposite for $\kappa<0$ (figure $11 b$ ). These conversions are most apparent after instability has grown to finite amplitude. There is also marked energy dissipation in the system and this 

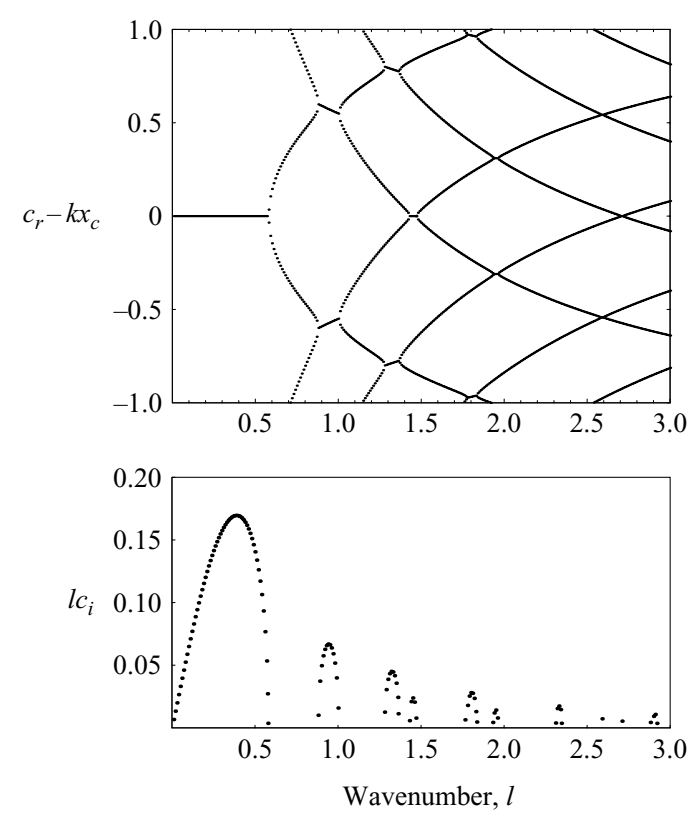

FIGURE 9. Similar to figure 5 but with $\kappa=-0.4$.

coincides with the onset of wave breaking. The energy conversions are consistent with the increase in the overall width of the flow, which for $\kappa>0$ means that dense fluid is flung up to higher elevation along the channel walls. This process may be regarded as a signature of barotropic instability, though this categorization must be reconciled with the property that there is no energy exchange between the mean flow and the growing wave, at least during the small-amplitude stage. Similarly, the increase in mean width for $\kappa<0$ is reminiscent of baroclinic instability: fluid at the edges descends to lower elevation and thereby loses potential energy.

Since the system suffers an energy loss and, as we will show, changes in potential vorticity, it is natural to ask how this occurs. The numerical algorithm has no explicit viscosity: it is a shock capturing code that has a level of intrinsic numerical dissipation that remains low except where regions of strong gradients are sensed (as near a hydraulic jump or bore). There the order of the scheme is reduced, resulting in an increase in the level of numerical dissipation. This increase is needed to control the numerical oscillations that typically arise near shocks. At the same time, the code ensures that the shocks contain no sources of mass or momentum. In an idealized shock (a discontinuity in depth and velocity) the solution can be calculated (and the rate of energy dissipation computed) from conservation of mass and momentum and is therefore independent of any explicit viscosity. In our simulations there are no obvious jumps or bores, but there are fine regions of strong gradients that form due to the breaking waves that occur around the edges of the flow. These features account for most of the energy dissipation.

An examination of the eigenfunction structure shows that the most unstable modes are trapped to the edges of the flow. In the case of the frontal wave instability, the modes decay monotonically inward from the edges. For instabilities involving inertiagravity waves, the modes oscillate but still decay inwards. The growth rate depends on the separation between the two edge regions. Readers familiar with the classical Eady (1949) model of baroclinic instability will see similarities with the present problem. 


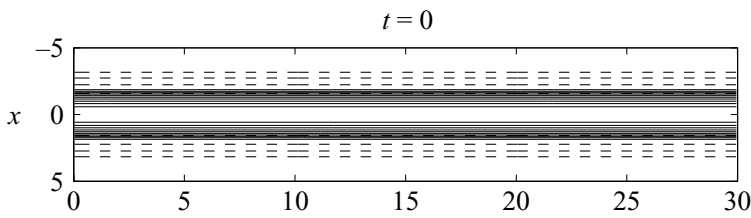

$t=60$

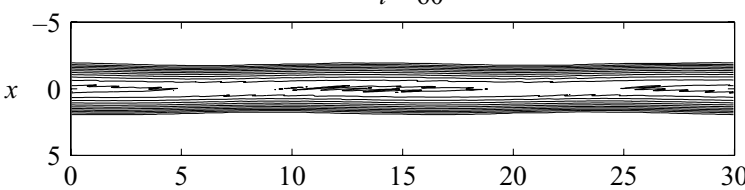

$t=80$

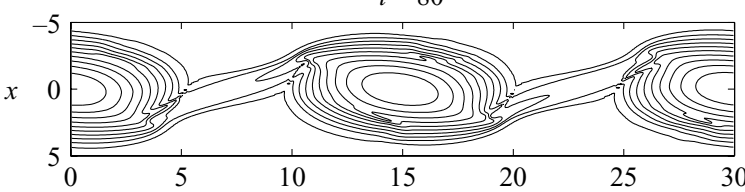

$t=100$

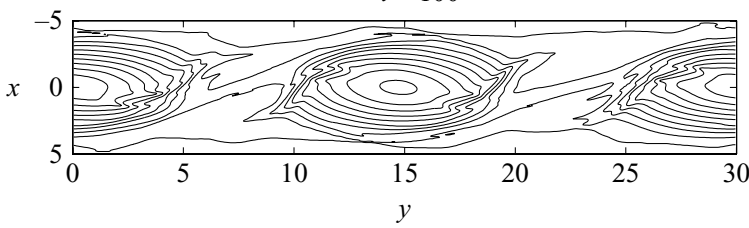

FIGURE 10. Similar to figure 6 but with $\kappa=-0.4$.
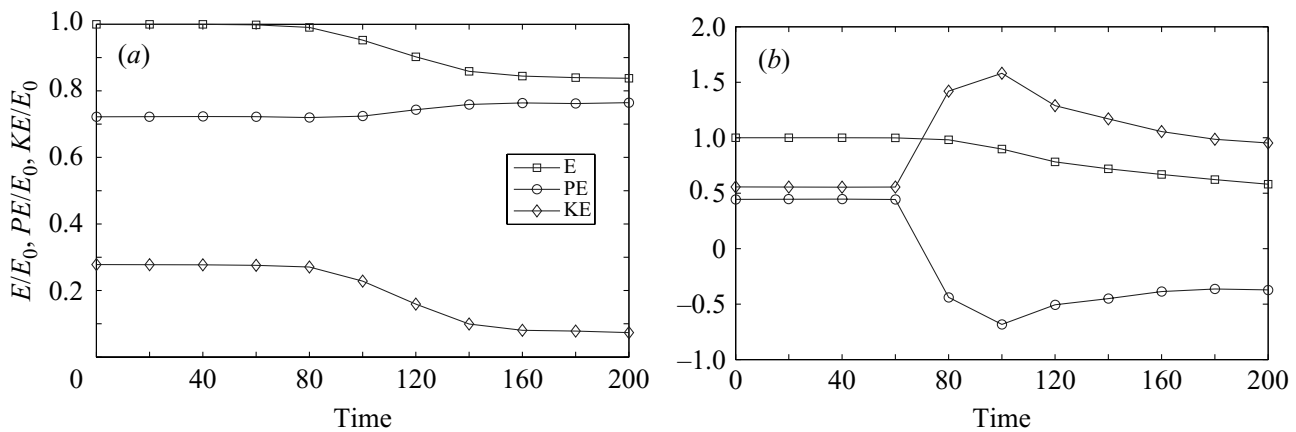

FIGURE 11. Total energy and its constituents $v$ s. time for $(a) \kappa=0.2$ and $(b) \kappa=-0.4$.

Both models involve edge waves that are separated by an interior region. (In the Eady problem, the 'edges' are rigid, horizontal, upper and lower boundaries.) The tendency of the waves is to propagate in opposite directions, but the sheared background flow can, over a certain range, bring the two speeds into equality. The waves then couple and experience resonant growth. The effect weakens as the upper and lower boundaries are separated.

\section{The equilibrated width and potential vorticity}

One measure of the finite-amplitude consequences of the instability is the ratio of the equilibrated width $w_{f}$ to the initial width $w_{o}$, plotted in figure 12 as a function 


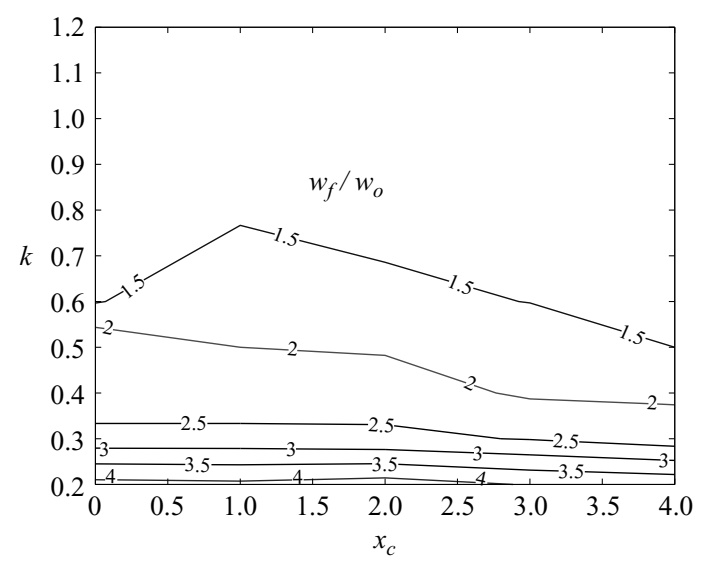

FIGURE 12. Contours of the ratio $w_{f} / w_{o}$ of final to initial current width as a function of $\kappa$ and of the initial value of $x_{c}$. The plot applies only to cases in which the flow equilibrates to a coherent, nearly parallel stream. (For $\kappa$ less than about 0.08 , the instability leads to a train of eddies.)

of $\kappa$ and of the initial value of $x_{c}$. The plot is based on 40 numerical experiments distributed over the parameter space shown. As suggested by the linear stability analysis, the results depend primarily on $\kappa$. More importantly, the results suggest that growth in the width of the current is significant only when $\kappa<1$; that is, when the channel is dynamically wide. When the channel width is moderate or narrow $(\kappa \geqslant O(1))$, the width of the flow remains close to the initial width and the zeropotential-vorticity approximation remains reasonable. Note that eddies are produced when $\kappa$ falls beneath a threshold value $\approx 0.08$.

What determines the final width $w_{f}$ ? A possibility is that the flow becomes sufficiently wide that the two edge waves lose contact with each other, as described above. A simple model of this effect can be formulated by assuming that the potential vorticity increases from zero in the initial state to a finite, uniform dimensional value $f / D_{\infty}$ in the equilibrated state. We will concentrate on cases for which $x_{c}$ is zero. Assuming that the edge waves decay over the deformation radius $\left(g^{\prime} D_{\infty}\right)^{1 / 2} / f$, the final width should be at least twice this value before contact between the two edge waves is lost. In particular let the final dimensional width equal $n\left(g^{\prime} D_{\infty}\right)^{1 / 2} / f$ where $n$ could reasonably be expected to lie the range $2-4$. A further constraint is provided by conservation of mass, which requires the cross-sectional area of the final state to equal that of the initial state. These areas can be calculated from the depth profile (2.8) for zero-potential-vorticity flow, and the non-dimensional depth profile

$$
D=\frac{1+\kappa}{q \sinh \left[q^{1 / 2} w\right]}\left\{\sinh \left[q^{1 / 2}\left(x-\frac{1}{2} w\right)\right]-\sinh \left[q^{1 / 2}\left(x+\frac{1}{2} w\right)\right]\right\}+\frac{1+\kappa}{q} w
$$

(e.g. Borenäs \& Lundberg 1986) for uniform, finite potential vorticity, nondimensionally $q=D / D_{\infty}$. Equating the initial and final areas leads to

$$
w_{f} / w_{o}=n\left[12\left(\frac{2(1-\cosh n)}{\sinh n}+n\right)\right]^{-1 / 3}
$$

and the corresponding $w_{f} / w_{o}$ are plotted for $n=2,3$ and 4 in figure $13 a$ along 

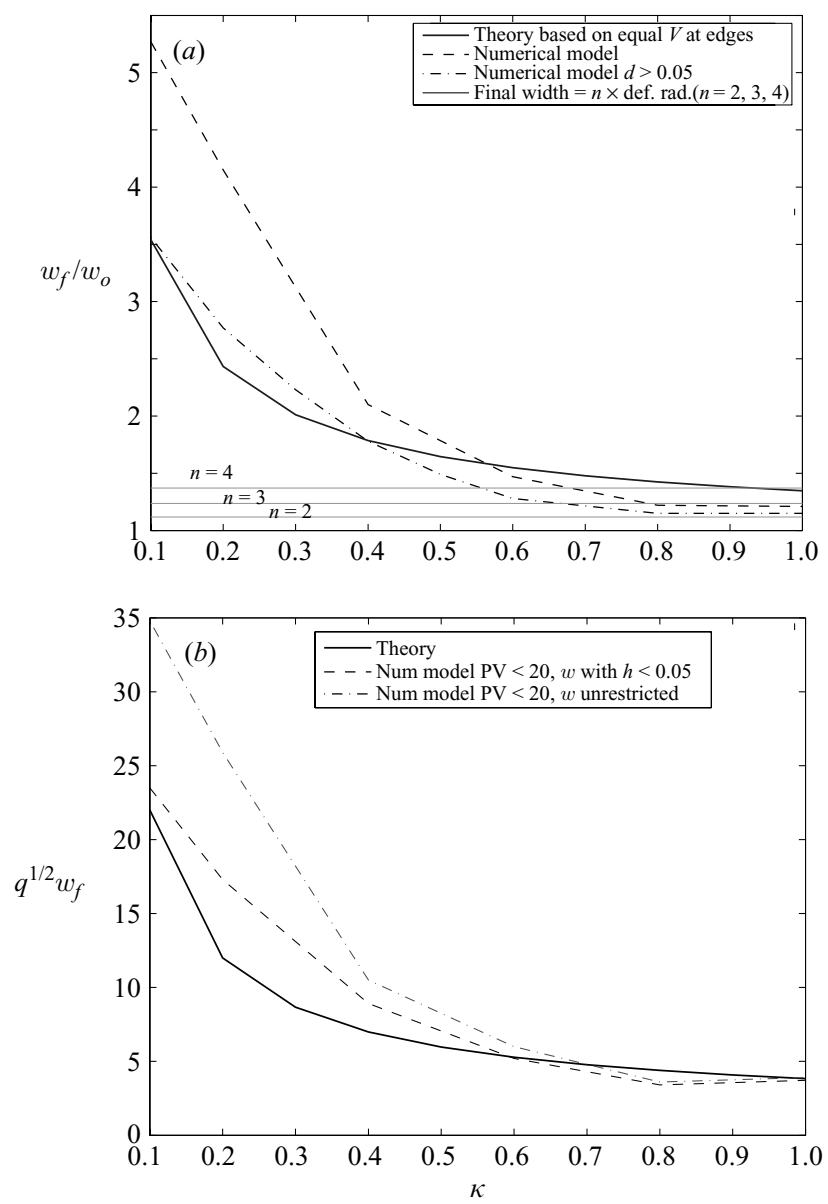

Figure 13. (a) Comparison between the numerical model values of $w_{f} / w_{o}$ (dashed curve) and a theory (equation (8.3), thick solid curve). The dot-dash curve gives the observed values if $w_{f}$ is based on depth greater than $5 \%$ of the maximum depth. The lower horizontal lines summarize a separate theory that requires the flow to equilibrate when its width grows to $n$ times the deformation radius. ( $b$ ) Comparison between the predicted equilibrated $q^{1 / 2} w$ based on (8.4) and the average value from the equilibrated numerical model solutions, obtained by neglecting values of $q$ less than 20. The dot-dashed line is based on the actual observed width while the dashed line uses the width based on layer depth values greater than 0.05 .

with the observed values for experiments with $x_{c}=0$. Aside from the fact that the prediction is independent of $\kappa$, the theory suffers from its severe underestimation of the observed $w_{f} / w_{o}$ over small values of $\kappa$. Clearly there is something more to the equilibration than just separation between the edge waves.

Alternatively, one might anticipate that the equilibration mechanism acts to satisfy Ripa's condition for stability. The theorem does not include decay scales for edge waves, but it does lay down the conditions under which wave pairs are unable to resonate. As we have already shown, a minimal requirement is that the fluid velocities at the two edges be equal. Since $V$ will be antisymmetrical about $x=0$ for $x_{c}=0$, this means that $V$ must vanish at both edges. Suppose then that we require the final state to have uniform potential vorticity, to have the same cross-sectional area as the initial state, and to have $V=0$ at the edges. Using the geostrophic velocity profile based on 
(8.1), namely

$$
V=\frac{1+\kappa}{q^{1 / 2} \sinh \left[q^{1 / 2} w\right]}\left\{\cosh \left[q^{1 / 2}\left(x-\frac{1}{2} w\right)\right]-\cosh \left[q^{1 / 2}\left(x+\frac{1}{2} w\right)\right]\right\}+\kappa x,
$$

one obtains

$$
\tanh \left[\sqrt{\frac{3}{1+\kappa}}\left(\frac{w_{f}}{w_{o}}\right)^{3 / 2}\right]=\frac{\kappa}{1+\kappa} \sqrt{\frac{3}{1+\kappa}}\left(\frac{w_{f}}{w_{o}}\right)^{3 / 2} .
$$

As shown in figure $13 a$ the prediction of $w_{f} / w_{o}$ based on (8.3) is an improvement in both trend and magnitude. For small values of $\kappa$ the theory underestimates the observed values and this may be due to the fact that the equilibrated numerical solutions typically have very thin and broad edge regions with anomalously high values of potential vorticity. (The values of $V$ at the edge tend to be greatly reduced from their initial values, though they are not zero.) If the thin edge regions are excised, which can be approximated by neglecting depth values less than 0.05 , the resulting data (dot-dashed curve) lie much closer to the theory. In any case, the results of this analysis suggest that the physical separation of waves in resonance is less important to the equilibration mechanism than is the avoidance of wave resonance altogether.

The theory leading to (8.3) also gives a prediction for the equilibrated potential vorticity. A convenient measure of this quantity is $q^{1 / 2} w_{f}$, which equals the ratio of the dimensional final width to the Rossby radius of deformation based on $D_{\infty}$. It follows from the above considerations that

$$
q^{1 / 2} w=2 \sqrt{\frac{3}{1+\kappa}}\left(\frac{w_{f}}{w_{o}}\right)^{3 / 2},
$$

where $w_{f} / w_{o}$ can be related to $\kappa$ using (8.3). As suggested in figure $13 b$, the resulting prediction does reasonably well when comparing the average potential vorticity of the numerical model. (The extremely high values of potential vorticity near the edges are ignored in the computation of this average, as explained in the figure caption.)

\section{The stability of a hydraulically controlled solution}

We now investigate the instability of a hydraulically controlled flow that originates in a deep upstream basin, passes over a shallow sill, and continues into a deep downstream basin, flowing along the right flank of the topography. The topography has $\kappa$ fixed at a value of 0.4 , with variable bottom height

$$
h_{o}(y)=\left(\exp \left\{-(\lambda y)^{2}\right\}-1\right)
$$

and $\lambda=0.125$. The steady solution, shown in the $t=0$ frame of figure 14 , is qualitatively similar to that shown in figure 3 . This state is what the steady hydraulic theory would predict, with a subcritical-to-supercritical transition taking place across the sill $(y=0)$. However, as shown at $t=100,120$ and 160, the flow undergoes the same instability and widening that has already been described, particularly in the subcritical region upstream of the sill $(y<0)$. Zero-potential-vorticity hydraulic theory clearly fails to provide a realistic view of the flow in this region. Open boundary conditions, with zero gradients in the along-channel direction, are used at the upstream and downstream edges of the numerical domain. In the case of the subcritical flow, the upstream boundary gives rise to a slight stabilization of the flow, as seen in figure 14 . The supercritical flow downstream of the sill is relatively free of disturbances, despite the fact that the linear growth rates are the same as for the upstream region $(\kappa$ being 

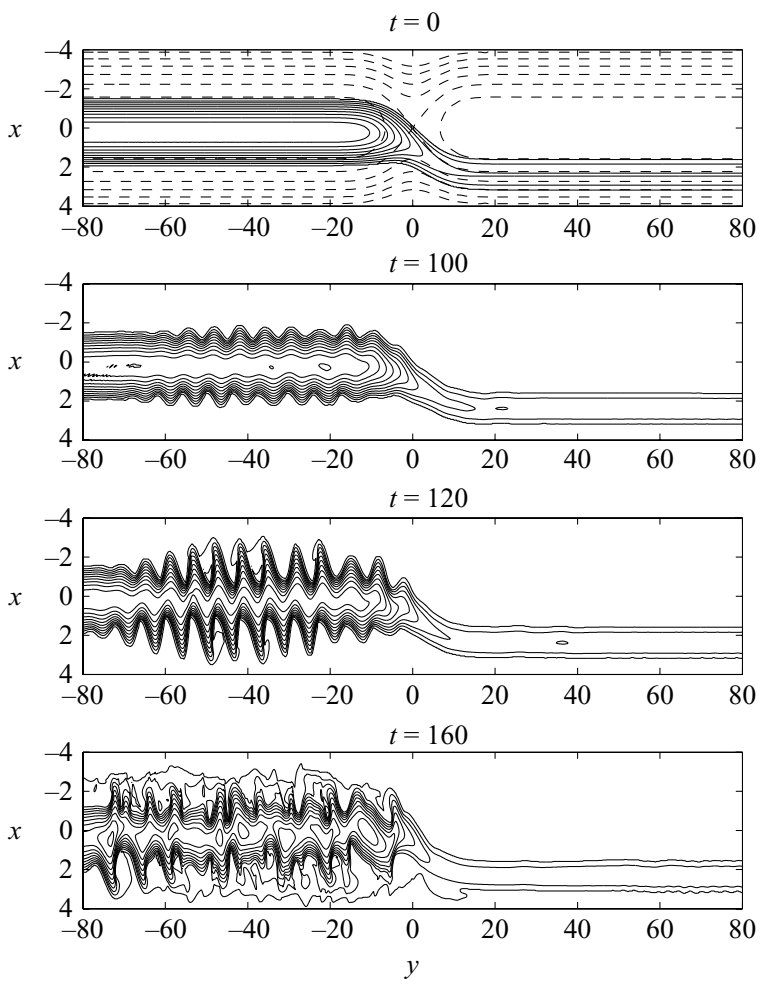

FIGURE 14. The instability of a hydraulically controlled flow in a channel with $\kappa=0.4$ and a sill (located at $y=0$.). The layer depth is contoured (solid lines) in intervals of 0.2 . The dashed lines show the topography contours in intervals of 0.5 .

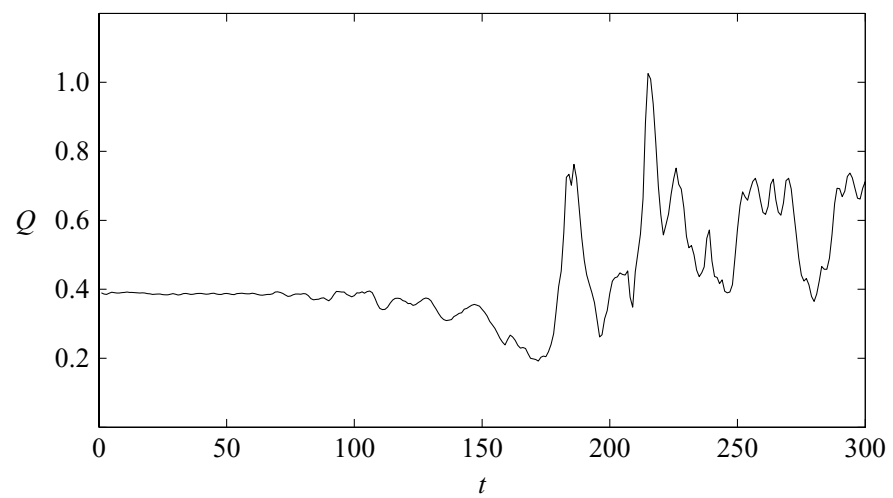

FIGURE 15. The volume flux $v$ s time for the flow shown in figure 14

the same). However, the phase speeds of the unstable modes are much greater in the supercritical portion and the waves apparently propagate downstream and out through the open boundary before they can grow to significant size.

The alteration of the initial flow is accompanied by a number of changes in bulk properties. For example, the volume flux at the sill diminishes during the early stages of evolution. A time history of the flux for the figure 14 example (figure 15) shows an initial decrease followed by large oscillations as the upstream waves reach the sill. 


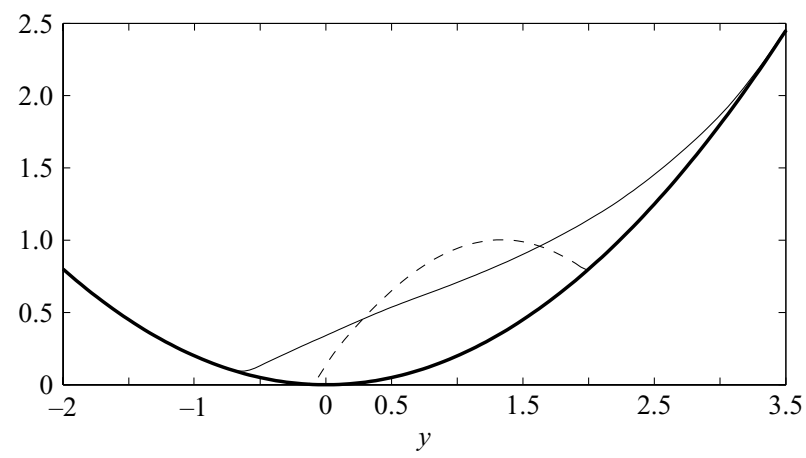

FIGURE 16. The sill flow at $t=0$ (dashed line) and the average conditions for $t=180-300$ (solid line) for the flow shown in figure 14.

The transport then increases, with a mean for $t=180-300$ of about 1.45 times the initial value. (These transport data are from a run identical to that shown in figure 14, but with a substantially longer upstream section so that the open upstream boundary does not affect the sill transport at the times shown.) Another striking change is that the region of counterflow at the sill is eliminated. The latter is demonstrated in figure 16, which shows cross-sectional interface height profiles at the sill at $t=0$ (dashed curve) and the average interface profile over the period $t=180-300$ (solid curve). The average is shown since the fluctuations during this period are very large; however, the average is characteristic of the instantaneous interface profiles during this period. Initially the interface near the right edge has a negative slope, indicating negative geostrophic velocities. At later stages, the slope and velocity are everywhere positive. In an apparent demonstration of the general conversion from potential to kinetic energy, fluid now lies further up on the right-hand slope of the topography. It is unclear whether the increase in the sill transport and the elimination of the counterflow will persist beyond the times shown owing to the uncertainties associated with the open upstream boundary. Resolution will require inclusion of a deep finite upstream basin.

The flow downstream of the sill does not produce the eddies that are observed in certain overflows. The lack of eddy generation seems consistent with previous models (e.g. Spall \& Price 1998 and Etling et al. 2000) that require a dynamically active overlying layer. However, it is possible to produce eddies in our model (figure 17) by lowering the curvature in the downstream region to the values (roughly $\kappa<.08$ ) that led to eddy formation in the uniform channel. This case shown is similar to that of figure 14 except the channel curvature is allowed to decrease from 0.4 at the sill to 0.004 in the downstream region. The topography and initial flow upstream of the sill are the same.

\section{Discussion}

The linear stability properties of zero-potential-vorticity flow in a parabolic channel depend only on the bottom curvature parameter $\kappa=2 \alpha g^{\prime} / f^{2}$, which can also be regarded as a dynamical measure of the channel width in a rotating system. Ripa's (1983) sufficient condition for stability can never be satisfied for such flows and our own eigenvalue calculations suggest that the flows are indeed unstable for finite $\kappa$. Maximum growth rates increase, as does the density of unstable wavenumbers, as the channel is made dynamically wider ( $\kappa$ is decreased). This trend continues 


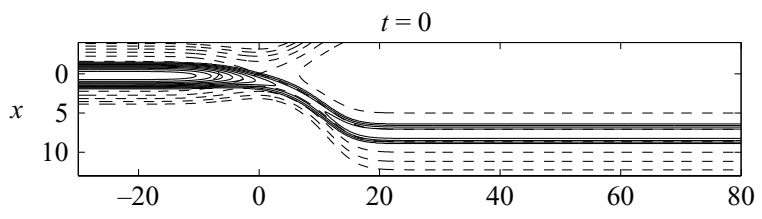

$t=40$

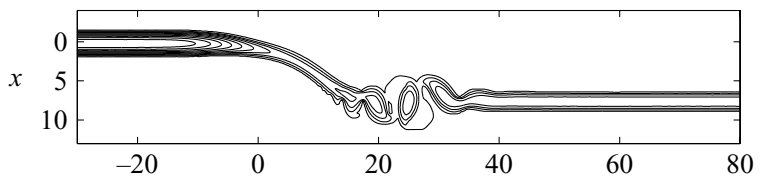

$t=120$

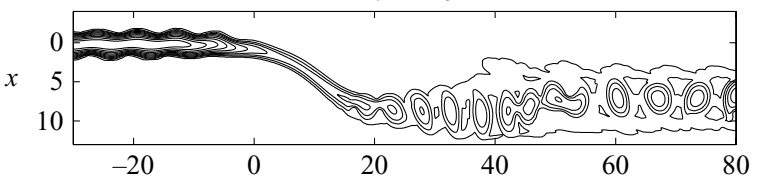

$t=160$

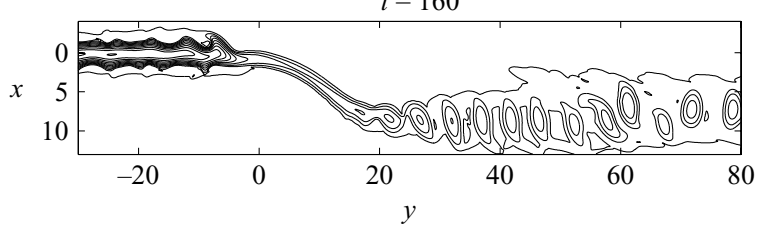

FiguRE 17. The instability of a hydraulically controlled flow in a channel with a sill (located at $y=0$ ), a channel curvature that is constant at $\kappa=0.4$ upstream of the sill and decreases to 0.04 downstream. Not all of the upstream numerical domain is shown. The layer depth is contoured (solid lines) in intervals of 0.2 . The dashed lines show the topography contours in intervals of 0.5 .

when the topography becomes convex ( $\kappa$ becomes negative). The largest growth rates are produced by a low-wavenumber, GKS-type instability, associated with the resonant interaction between two waves trapped to opposite edges of the stream. Other bands of instability associated with inertia-gravity wave resonances also exist at high wavenumber, but the growth rates are smaller. As the channel narrows $(\kappa$ increases), all the resonant bands migrate to higher wavenumber and the maximum growth rates diminish. (Stern \& Simeonov (2008) have shown that the growth rate goes to zero as $\kappa \rightarrow \infty$ for all finite wavenumbers.)

At finite amplitude, the instability leads to wave breaking at the stream edges. For $\kappa<0.08$ the flow eventually breaks into a train of eddies. There is some evidence for this type of behaviour in the southern reaches of the deep western boundary layer in the North Atlantic ocean (Dengler et al. 2004). With $\kappa>0.08$, wave breaking still occurs, but the overall effect is to widen the flow into a coherent stream. The linearly unstable disturbances extract no energy from the mean flow and this makes it difficult to classify the instability as barotropic or baroclinic. However, there is a general conversion from kinetic to potential energy for $\kappa>0$, a trend that is most evident as the wave grows to large amplitude. The increase in potential energy is caused by an increase in the average width of the flow, causing fluid to be flung up to higher topographic elevations. The energy conversion is reversed for $\kappa<0$; there the same widening causes fluid at the edges to be moved to lower elevation. 
One measure of the finite-amplitude consequences of the instability is the ratio $w_{f} / w_{o}$ of equilibrated width to initial width. The numerical results suggest that $w_{f} / w_{o}$ depends primarily on $\kappa$ and only weakly on the initial value of $x_{c}$. For small $\kappa, w_{f} / w_{o}$ can be as large as 4 or 5 , but the ratio decreases and rapidly approaches unity as $\kappa$ grows beyond unity. Thus, the zero-potential-vorticity approximation remains reasonable for channels with $\kappa \geqslant O(1)$. We have explored the equilibration mechanism by means of two simple models that attempt to predict $w_{f} / w_{o}$. Both assume that the potential vorticity of the equilibrated state can be approximated by a finite constant, dimensionally $f / D_{\infty}$ and non-dimensionally $q$. In the first model, resonant edge waves are assumed to decay over a scale $\left(g^{\prime} D_{\infty}\right)^{1 / 2} / f$ and therefore lose contact with each other when $w_{f}=n\left(g^{\prime} D_{\infty}\right)^{1 / 2} / f$, where $n$ plausibly lies in the range 2-4. Attractive as this idea is, the resulting prediction fails to depend on $\kappa$ and underestimates the $w_{f} / w_{o}$ values observed for small $\kappa$ by an order of magnitude. In the second model, the prediction is based on the idea that Ripa's theorem is satisfied by the equilibrated flow. This requires, at the very least, that the fluid velocities at the two edges be equal. For a flow that is symmetric (in depth) about $x=0$, the edge velocities must be zero. The resulting model is much more reasonable in that the $w_{f} / w_{o}$ prediction decreases with increasing $\kappa$ and matches the numerical model values within a factor of about 2 . Among the possible reasons the agreement is not closer is that the potential vorticity of the equilibrated state is not constant, but shows anomalously high values near the edges. In some cases the edges themselves are very thin and broad.

In studies using zero potential vorticity, realistic bottom topography and uniform potential vorticity (e.g. Nikolopoulos et al. 2003; Borenäs \& Nikolopoulos 2000) the hydraulic model sill flow often has a band of counterflow at the right-hand edge. This feature is judged as being unrealistic and the counterflow is sometimes disregarded in the calculation of the volume flux. The resulting flux estimate, which is based only those portions of the flow having positive velocity, can be considerably greater than the total flux of the model stream. We have found that the instability eliminates the reverse flow, which is in general agreement with observations from places like the Faroe-Bank Channel (e.g. Mauritzen et al. 2005 and Girton et al. 2006). The volume flux initially decreases, but then recovers and becomes highly variable. In order to calculate a time-average flux and compare it with what is predicted by hydraulic theory, it is probably necessary to extend the numerical channel into a finite upstream basin and consider the dynamics of this basin it its entirety.

The value $\kappa \approx 2$ can be estimated for the sill section in the Faroe-Bank Channel based on a parabolic fit the topography (Girton et al. 2006). Although the sill section in the Denmark Strait is less like a parabola, it is considerably wider than the deformation radius. The nominal value of $\kappa$ is probably an order of magnitude smaller. It is not surprising then, that the flow there has been observed to be more highly variable than at the Faroe-Bank Channel sill (e.g. Hansen, Turnbull \& Qsterhus 2001).

This work was supported by the National Science Foundation (Grant OCE0525729). The authors wish to thank J. Simeonov and M. Stern for a number of helpful discussions.

BorENÄs, K. M. 1988 Some aspects of rotating channel flow. PhD Thesis, University of Goteborn, Chapter 4. 
Borenäs, K. M. \& Lundberg, P. A. 1986 Rotating hydraulics of flow in a parabolic channel. J. Fluid Mech. 167, 309-326.

Borenäs, K. M. \& LundberG, P. A. 1988 On the deep-water flow through the Faroe Bank Channel. J. Geophys. Res. 93(C2), 1281-1292.

Borenäs, K. M. \& Nikolopoulos, A. 2000 Theoretical calculations based on real topography of the maximal deep-water flux through the Jungfern Passage. J. Marine Res. 58, 709-719.

Bruce, J. G. 1995 Eddies southwest of the Denmark Strait. Deep-Sea Res. 42, 13-29.

Charney, J. G. \& Stern, M. 1962 On the stability of internal baroclinic jets in a rotating atmosphere. J. Atmos. Sci. 19, 159-172.

Dengler, M., Schott, F. A. Eden, C. Brandt, P. Fischer, J. \& Zantopp, R. J. 2004 Break-up of the Atlantic deep western boundary current into eddies at $8^{\circ} \mathrm{S}$. Nature 432, 1018-1020.

Dickson, R. R. \& Brown, J. 1994 The production of North Atlantic Deep Water: sources, rates and pathways. J. Geophys. Res. 99, 12319-12341.

EADY, E. T. 1949 Long waves and cyclone waves. Tellus 1, 33-52.

Etling, D. Gelhardt, F. Schrader, U. Brennecke, F. Kuhn, G. Chabert D’Hieres, B. \& Didelle, H. 2000 Experiments with density currents on a sloping bottom in a rotating fluid. Dyn. Atmosp. Oceans 31, 139-164.

Farrell, B. F. \& IoAnnou, P. J. 1996 Generalized stability theory. Part I: Autonomous operators. J. Atmos. Sci. 53, 2025-2040.

FJøTORFт, R. 1950 Application of integral theorems in deriving criteria of stability for laminar flows and for the baroclinic circular vortex. Geophys. Publ. 17, 1-52.

Gill, A. E. 1977 The hydraulics of rotating-channel flow. J. Fluid Mech. 80, 641-671.

Girton, J., Pratt, L. Sutherland, D. \& Price, J. 2006 Is the Faroe Banck Channel overflow hydraulically controlled? J. Phys. Oceanogr. 36, 2340-2349.

Griffiths, R. W., Killworth, P. D. \& Stern, M. E. 1982 Ageostrophic instability of ocean currents. J. Fluid Mech. 117, 343-377.

Hansen, G., Turnbull, W. R. \& Østerhus, S. 2001 Decreasing overflow from the Nordic Seas through the Atlantic Ocean through the Faroe Bank Channel since 1950. Nature 411, 927-930.

HaYashi, Y.-Y. \& Young, W. R. 1987 Stable and unstable shear modes of rotating parallel flows in shallow water. J. Fluid Mech. 184, 477-504.

Helfrich K. R., Kuo, A. \& Pratt, L. J. 1999 Nonlinear Rossby adjustment in a channel. J. Fluid Mech. 390, 187-222.

Helfrich K. R. \& Pratt, L. J. 2003 Rotating hydraulics and upstream basin circulation. J. Phys. Oceanogr. 33, 1651-1633.

Howard, L. N. 1961 Note on a paper of John W. Miles. J. Fluid Mech. 10, 509-512.

Killworth, P. D. 1992 Flow properties in rotating, stratified hydraulics. J. Phys. Oceanogr. 22, 997-1017.

Kuo, H. L. 1949 Dynamic instability of two-dimensional nondivergent flow in a barotropic atmosphere. J. Meter. 6, 105-122.

Mauritzen, C. Price, J. Sanford, T. \& Torres, D. 2005 Circulation and mixing in the Faroese Channels. Deep-Sea Res. I, 52, 883-913.

Nikolopoulos, A., Borenäs, K. M. Hietala, R. \& Lundberg, P. 2003 Hydraulic estimates of Denmark Strait overflow. J. Geophys. Res. 108, 3095, doi:10.1029/2001JC001283.

Paldor, N. 1983 Stability and stable modes of coastal fronts. Geophys. Astrophys. Fluid Dyn. 27, 217-218.

Pratt, L. J., Helfrich, K. R. \& Chassignet, E. P. 2000 Hydraulic adjustment to an obstacle in a rotating channel. J. Fluid Mech. 404, 117-149.

Pratt, L. J. \& Whitehead, J. A. 2008 Rotating Hydraulics. Springer.

RaYleigh, LORD. 1880 On the stability, or instability of certain fluid motions. Proc. Lond. Math. Soc. 9, 57-70.

RIPA, P. 1983 General stability conditions for zonal flows in a one layer model on a beta-plane or the sphere. J. Fluid Mech. 126, 463-487.

Satomura, T. 1981a. An investigation of shear instability in a shallow water. J. Met. Soc. Japan 59, 148-167.

SATOMURA, T. $1981 b$ Supplementary note on shear instability in a shallow water. J. Met. Soc. Japan 59, 168-171. 
Shen, C. Y. 1981 The rotating hydraulics of open-channel flow between two basins. J. Fluid Mech. 112, $161-188$.

Smith, P. C. 1976 Baroclinic instability in the Denmark Strait Overflow. J. Phys. Oceanogr. 6, 355-372.

Spall, M. A. \& Price, J. F. 1998 Mesoscale variability in Denmark Strait: the PV outflow hypothesis. J. Phys. Oceanogr. 28, 1598-1623.

Stern, M. E. \& Simeonov, J. A. 2008 Frontal waves in a strait. J. Fluid Mech. 598, 321-334.

SwAters, G. E. 1991 On the baroclinic instability of cold-core coupled density fronts on a sloping continental shelf. J. Fluid Mech. 224, 361-382.

Whitehead, J. A., Leetma, A. \& Knox, R. A. 1974 Rotating hydraulics of strait and sill flows. Geophys. Fluid Dyn. 6, 101-125.

Worthington, L. V. 1969 An attempt to measure the volume transport of Norwegian Sea overflow water through the Denmark Strait, Deep-Sea Res. 16 (supp) 421-432. 